\title{
Creencias implícitas y orientaciones de meta de jugadoras mexicanas de sóftbol elite Implicit beliefs and goal orientations in Mexican elite softball players
}

\author{
*Jorge Zamarripa, **Manuel Francisco De la Cruz Ortega, ***Octavio Álvarez, ***Isabel Castillo \\ *Universidad Autónoma de Nuevo León (México), ${ }^{* *}$ Universidad Estatal de Sonora (México), ***Universidad de Valencia (España)
}

\begin{abstract}
Resumen. Dentro del contexto deportivo, las personas pueden presentar dos tipos de creencias implícitas sobre la habilidad deportiva, creencias incremental y de entidad, las cuales tienen un papel importante en la conformación de las orientaciones de meta cuando practican deporte. Hasta la fecha no se conocen estudios con población mexicana que hayan examinado la relación entre las creencias implícitas y las orientaciones de meta. El objetivo del presente estudio consistió en examinar las creencias implícitas sobre la habilidad y las orientaciones de meta en una muestra de jugadoras de sóftbol de máximo nivel competitivo de México. La muestra estuvo compuesta por 71 jugadoras de sóftbol $\left(M_{\text {edad }}=22.05\right.$ años; $D T=6.27 ;$ rango = 13-41) pertenecientes a las selecciones de seis estados de la República Mexicana. Los resultados revelaron buena consistencia interna de los instrumentos. Las jugadoras presentaron una alta creencia incremental y una alta orientación a la tarea. La creencia incremental correlacionó positivamente con la orientación a la tarea, y ésta a su vez con la creencia estable pero de manera negativa. Por su parte, la creencia de entidad correlacionó positivamente con la orientación al ego. La creencia incremental de habilidad percibida predijo la orientación a la tarea y la creencia de entidad predijo la orientación al ego. Se deben desarrollar creencias de que la habilidad se puede mejorar a través del esfuerzo dado las consecuencias positivas que tiene en el fomento de una percepción de competencia orientada a la tarea y ésta a su vez sobre las experiencias deportivas. Palabras clave: Deporte, México, metas de logro, habilidad, motivación.
\end{abstract}

Abstract. In sport contexts, people can display two different types of implicit beliefs about athletic skills: incremental beliefs and entity beliefs, both playing an important role in setting a particular goal orientation while practicing any sport. There are no studies that examine the relationship between implicit beliefs and goal orientations in Mexican populations so far. The aim of this study was to analyze implicit beliefs about athletic skills and goal orientations in a sample of Mexican high performance softball players. The sample was composed by 71 softball players $\left(M_{\text {age }}=22.05\right.$ years; $S D=$ 6.27; range $=13-41$ ) from selection teams of six states of the Mexican Republic. Preliminary analyses demonstrated good internal consistency of the instruments applied. Players showed high incremental beliefs as well as high task orientation. These variables were also found to be positively correlated. In addition, task orientation was negatively associated with stable beliefs. On the other hand, entity beliefs were positively related to ego orientation. Incremental beliefs predicted task orientation, whereas entity beliefs predicted ego orientation. As our results suggest, we need to foster the belief that skills can be improved through sacrifice, as it would prompt the perception of task-oriented competence, thus having a positive impact on sports experiences.

Keywords: Sport, Mexico, achievement goals, ability, motivation.

\section{Introducción}

En el deporte, así como en otros contextos de logro como el laboral y académico, los objetivos o metas son considerados como esenciales para el buen funcionamiento y tienen un papel decisivo en el fomento de la motivación, y el rendimiento (Hagger \& Chatzisarantis, 2005). Asimismo, las creencias implícitas sobre si las habilidades humanas son fijas o maleables son consideradas predictoras de la motivación, la diversión, la autoeficacia, la persistencia y los afectos positivos entre otros (Biddle, Wang, Chatzisarantis \& Spray 2003). Dado los beneficios asociados a la práctica deportiva, resulta de gran interés el estudio de las metas predominantes en el contexto deportivo así como de su relación con las creencias implícitas sobre la habilidad deportiva (Organización Mundial de la Salud, 2010).

Desde la Teoría de las Metas de Logro (Nicholls, 1989) se ha propuesto que existen al menos dos orientaciones de meta o formas de juzgar la competencia y por el que subjetivamente se definen el éxito y el fracaso en los contextos de logro, denominadas orientación a la tarea o a la maestría y orientación al ego o al resultado, asociadas con respuestas motivacionales adaptativas o desadaptativas (e.g., alto o bajo esfuerzo, alta o baja implicación, persistencia o abandono de la actividad). Estas orientaciones están relacionadas con la forma en las que las personas interpretan el éxito y el fracaso (Nicholls, 1989). Es decir, que las personas que buscan demostrar competencia y conseguir una meta en situaciones de logro (i.e., un deportista), difieren del reconocimiento que estos se le den al éxito y al fracaso, en otras palabras, lo que significa éxito para uno, puede interpretarse como fracaso para otro.

En la orientación de meta a la tarea, la visión de las perso-

Fecha recepción: 05-01-16. Fecha de aceptación: 20-05-16 Jorge Zamarripa

jorge.zamarriparv@uanl.edu.mx nas tiende a ser más personal o autoreferenciada. Las percepciones de una alta habilidad y los éxitos subjetivos se basan en las experiencias de aprendizaje, en la ejecución personal y el dominio de la tarea (Nicholls, 1989). Por lo tanto, los deportistas que tienen una orientación a la tarea perciben el deporte como una actividad que fortalece la capacidad de cooperación, la responsabilidad social y la motivación intrínseca, y a la vez fortalece los afectivos positivos (Chin, Knoo \& Low, 2012; Duda, Chi, Newton, Walling \& Catley, 1995; Duda, 2012; Ntoumanis \& Biddle, 1999). Muestran un incremento en el interés por aprender, esforzarse al máximo, mejorar su salud, cumplir con el reglamento del deporte en el cual se participa y mejorar sus habilidades dentro de este deporte identificando el éxito cuando se demuestra el dominio y siendo el esfuerzo el mayor éxito (Parish \& Treasure, 2003; Standage \& Treasure, 2002).

En la orientación al ego, o hacia uno mismo, los sentimientos de logro personal y alta competencia están referidos a las normas externas. Para ellos, la demostración de la habilidad se basa en hacerlo mejor que los otros con el mínimo esfuerzo (Nicholls, 1989). Los deportistas que tienen una orientación al ego (o al resultado) buscan demostrar que tienen un mayor nivel de competencia que los demás compañeros o rivales dentro de su deporte con igual o menos esfuerzo, es decir, estos deportistas sólo se sienten con éxito cuando creen que su desempeño de manera individual fue mejor que el desempeño de los otros en la misma competición. Estos a su vez pretenden que el deporte les ayude a obtener un mayor reconocimiento o estatus social, desean ser más populares, todo esto realizando el mínimo esfuerzo posible, estableciendo relaciones mínimas con la motivación intrínseca hacia la propia actividad (Ames, 1992; Dweck 1986; Duda, 2005; Duda \& Whitehead, 1998; Nicholls 1984; 1989).

Las creencias implícitas son creencias adquiridas sobre la naturaleza de las habilidades humanas que ejercen una fuerte influencia sobre las conductas, ya que proporcionan un marco 
subyacente para la interpretación y respuesta a los eventos dentro de los contextos de logro (Dweck \& Leggett, 1988). Hay dos tipos de creencias implícitas: la creencia incremental y las creencias de entidad. Aquellos que consideran un atributo como algo estable, reflejan una creencia de entidad o fija, mientras que aquellos que piensan que dicho atributo es modificable o moldeable muestran una creencia incremental (Dweck, 1999).

Los estudios en el contexto educativo, han encontrado que las creencias implícitas que los estudiantes tienen sobre ciertos atributos (i.e., la inteligencia), tienen un papel importante en la conformación de las orientaciones de meta (Dweck, 1999; Dweck \& Leggett, 1988). Según Dweck, Chiu y Hong (1995), las creencias de entidad e incrementales son percepciones específicas de contextos específicos, por lo tanto, las creencias sobre los atributos como la inteligencia que son más comunes en el contexto educativo, no tienen por qué estar relacionadas con las creencias que una persona tenga sobre su habilidad en el deporte.

En este sentido, dentro del contexto deportivo y al igual que en otros contextos, se han establecido dos tipos de creencias implícitas sobre la habilidad deportiva, una creencia de entidad o fija y una creencia incremental o modificable. Una creencia de entidad considera la habilidad como algo estable, y por lo tanto, independientemente del esfuerzo y la práctica, ésta no puede modificarse, y la actuación depende de capacidades innatas. Por su parte, una creencia incremental implica que la habilidad puede ser mejorada con un incremento del esfuerzo, el aprendizaje y la práctica (Biddle, Soos \& Chatzisarantis, 1999; Biddle et al., 2003; Nicholls, 1992; Sarrazin, Biddle, Famose, Cury, Fox \& Durand, 1996). Diversas investigaciones realizadas en el contexto deportivo han mostrado una asociación entre las creencias implícitas sobre la habilidad deportiva y la motivación, la diversión, la persistencia, la auto-eficacia, los afectos positivos y las orientaciones de meta (e.g., Biddle et al., 2003; Jourden, Bandura \& Banfield, 1991; Van-Yperen \& Duda, 1999).

En una serie de estudios realizados por Sarrazin et al. (1996) estudiaron las relaciones entre las creencias de habilidad y las orientaciones de meta en una muestra de 498 niños que participaban en algún deporte (194 niños de 11 y 12 años de edad [ $M$ y $D T$ no reportado] del sureste de Inglaterra y 304 niños de 11 a 17 años $[M=14.0 ; D T=1.62]$ de Paris, Francia). Los resultados revelaron relaciones positivas entre la creencia de que la habilidad deportiva es una entidad, un regalo natural y es general con la orientación al ego; mientras que encontraron asociaciones positivas entre la creencia de que la habilidad es incremental, producto del aprendizaje y es específica con la orientación a la maestría, así como relaciones negativas entre dicha orientación a la maestría con la creencia de que la habilidad es estable y un regalo natural.

Por su parte, King y Williams (1997) examinaron las relaciones entre las orientaciones de meta, el éxito y las creencias implícitas de habilidad y rendimiento en 68 estudiantes novatos de artes marciales de 17 a 45 años de edad $(M=22.90 ; D T=$ 5.8). Los resultados de este estudio revelaron una relación marginalmente positiva entre la orientación al ego y la creencia de que la habilidad deportiva es estable. Por el contrario, la orientación hacia la tarea se relacionó negativa y significativamente con las creencias de que la habilidad es estable y con la creencia de que la habilidad es un talento natural.

Asimismo, Fonseca y Paula Brito (2000), examinaron las relaciones entre las concepciones acerca de la naturaleza de la competencia deportiva y los objetivos de logro en dos muestras de individuos con diferentes relaciones con el deporte. En el primer estudio participaron 498 deportistas federados de ambos sexos con edades entre los 12 y los 33 años $(M=17.65$; $D T$ $=3.60$ años). En el segundo estudio participaron 576 estudiantes con edades entre los 12 y los 17 años que practicaban depor- te pero no de modo competitivo. Los resultados revelaron que los deportistas que creían en mayor medida que la habilidad deportiva es una consecuencia del aprendizaje, mejorable, y específico estaban más orientados a la tarea. Por el contrario, los deportistas que creían en mayor medida que la habilidad deportiva es un talento natural, estable, y general, tendían a estar más orientados al ego.

Por su parte, Biddle et al. (2003) examinaron las relaciones entre las creencias de habilidad deportiva, las orientaciones de meta, la percepción de competencia y la no motivación (amotivation) en una muestra de 2969 estudiantes ingleses (1566 chicas y 1453 chicos) de 11 a 14 años de edad $(M=12.9 ; D T=$ $0.9)$. Los resultados revelaron que la creencia de habilidad deportiva incremental predijo la orientación hacia la tarea y la creencia de habilidad deportiva de entidad predijo la orientación al ego.

Hasta el momento no se conocen estudios realizados con población mexicana donde se hayan examinado la relación entre las creencias implícitas sobre la habilidad deportiva y las orientaciones de meta en el contexto deportivo, tampoco se conocen estudios que hayan examinado las diferencias entre estas variables en función de la edad y los años de experiencia. Por lo tanto, el objetivo del presente estudio consiste en examinar las creencias implícitas sobre la habilidad y las orientaciones de meta en jugadoras de sóftbol de máximo nivel competitivo de México.

\section{Método}

\section{Participantes}

La muestra estuvo compuesta por 71 jugadoras de sóftbol $\left(M_{\text {edad }}=22.05\right.$ años; $D T=6.27$; rango $\left.=13-41\right)$ que pertenecen a las selecciones de los estados de Yucatán $(n=11,15.5 \%)$, Sinaloa $(n=11,15.5 \%)$, Baja California Sur $(n=10,14.1 \%)$, Sonora ( $n=12,16.9 \%)$, Chihuahua $(n=15,21.1 \%)$ y Baja California Norte ( $n=12,16.9 \%$ ) y que participaron en el campeonato nacional de sóftbol femenil 2015, categoría primera fuerza. La selección de la muestra se llevó a cabo través de un muestreo no probabilístico, intencional de conveniencia.

La mayoría de las jugadoras se sitúan entre los 18 y los 24 años de edad (36.6\%), seguido de aquellas con un rango de edad entre los 25 y los 41 años (33.8\%) y aquellas de edades entre los 13 y los 17 años (29.6\%). La mayoría tenían entre seis y diez años de experiencia (36.6\%), seguido de aquellas con más de diez años (35.2\%) y el porcentaje menor de jugadores lo componen aquellas con cinco años o menos de experiencia (28.2\%). El promedio de años practicando el sóftbol fue de 9.76 años de experiencia $(D T=5.97 ;$ rango $=2-32$ años $)$.

\section{Instrumentos}

Creencias implícitas de habilidad. Se utilizó la versión española (Moreno-Murcia, Cervelló-Gimeno, Martínez-Galindo \& Moreno, 2013) del Cuestionario de las Concepciones de la Naturaleza de la Habilidad Deportiva-2 (Biddle et al., 2003). El cuestionario está compuesto por 12 ítems divididos en dos subescalas de orden superior denominadas creencia incremental y creencia de entidad. Los ítems están precedidos por la frase: «Tus creencias sobre tu habilidad en deporte son...». La subescala de creencias de entidad está compuesta por seis ítems, de los cuales tres corresponden a la variable estable de primer orden y los otros tres a la variable talento. Por su parte, la subescala incremental está formada por seis ítems, de los cuales tres corresponden a la variable mejora de primer orden y los otros tres a la variable aprendizaje. Un ejemplo de ítem de creencia de entidad es «Necesitas tener algún «talento natural» para ser buena en deporte» y un ejemplo de creencia incremental es «Si te esfuerzas lo suficiente, siempre conseguirás mejorar en 
deporte». Las respuestas se recogen mediante una escala tipo Likert de cinco puntos que oscila desde totalmente en desacuerdo (1) a totalmente de acuerdo (5). Las propiedades psicométricas de la versión al castellano utilizada por Moreno-Murcia et al. (2013) ha presentado una adecuada consistencia interna con valores alfa de .87 para el factor entidad, de .72 para la variable estable y de .86 para el talento. Por su parte, el valor alfa del factor incremental fue de .83, de .68 para la mejora y .73 para el aprendizaje.

Orientaciones de meta. Se utilizó la versión adaptada al contexto mexicano (López-Walle, Balaguer, Meliá, Castillo \& Tristán, 2011) del Cuestionario de Orientación al Ego y la Tarea en el Deporte (Duda, 1989). El cuestionario consta de 13 ítems divididos en dos subescalas: orientación a la tarea y orientación al ego. Dichos ítems vienen precedidos por la frase: «Yo siento que tengo más éxito en el sóftbol cuando...». Un ejemplo de ítem de la orientación a la tarea es «Aprendo una nueva habilidad esforzándome mucho» y un ejemplo de ítem de la orientación al ego es «Las otras no pueden hacerlo tan bien como yo». Las respuestas se recogen mediante una escala tipo Likert de cinco puntos que oscila desde muy en desacuerdo (1) a muy de acuerdo (5). La fiabilidad de esta versión del instrumento reportada en el estudio de López-Walle et al. (2011) mostró valores adecuados (alfas de .85 para ambos factores).

\section{Procedimiento}

La aplicación de los instrumentos se llevó a cabo de manera autoadministrada con el consentimiento previo de las jugadoras y del entrenador responsable de cada equipo. Las participantes fueron informadas del objetivo del estudio, de la voluntariedad, absoluta confidencialidad de las respuestas y manejo de los datos, que no había respuestas correctas o incorrectas y se les solicitó que respondieran con la máxima sinceridad y honestidad. Todas las jugadoras accedieron a participar en el estudio tardando diez minutos en promedio en contestar la información solicitada. A las jugadoras menores de edad se les solicitó el permiso informado de los tutores legales.

\section{Resultados}

\section{Consistencia interna de los instrumentos}

La consistencia interna de los dos instrumentos se analizó a través del estadístico alfa de Cronbach (1951). Debido a que las correlaciones ítem-total fueron altas y que la eliminación de ningún ítem mejoraba los coeficientes de fiabilidad, se mantuvieron los doce ítems del Cuestionario de las Concepciones de la Naturaleza de la Habilidad Deportiva-2 y los trece ítems del Cuestionario de Orientación al Ego y la Tarea en el Deporte. Los coeficientes alfa de las distintas dimensiones de ambos cuestionarios fueron satisfactorios (véase diagonal de la Tabla $1)$.

\section{Estadísticos descriptivos}

En la Tabla 1 se muestran las medias y desviaciones típicas de las orientaciones de meta (orientación al ego y orientación a la tarea), los tipos de creencias (creencia de entidad y creencia incremental) y las variables de primer orden de dichas creencias (estable, talento, mejora y aprendizaje). En general, las jugadoras de sóftbol presentaron una mayor concepción de que la habilidad es de tipo incremental en comparación con la creencia de que la habilidad es de tipo entidad $(t=14.26, p<.001)$. Las jugadoras consideran que su habilidad viene determinada principalmente por el aprendizaje y es mejorable, siendo en menor medida la creencia de que su habilidad deportiva es un talento natural y estable. Por otro lado, las jugadoras mostraron una alta orientación hacia la tarea, la cual fue mayor que su orientación al ego $(t=16.07, p<.001)$.
Diferencias de las variables del estudio según los equipos, grupos de edad y años de experiencia

Para conocer si existían diferencias por equipos, grupos de edad y años de experiencia entre las orientaciones de meta (orientación al ego y orientación a la tarea) y las variables de orden superior (creencia de entidad y creencia incremental) y primer orden (estable, talento, aprendizaje y mejora) de las creencias de habilidad se realizaron diferentes análisis de varianza. Los resultados no revelaron diferencias significativas en ninguna de las variables analizadas.

\section{Correlación entre las variables del estudio}

Tal como se muestra en la Tabla 1, la correlación entre la orientación a la tarea y la orientación al ego no resultó significativa, al igual que la correlación entre la creencia de que la habilidad es una entidad y la creencia de que dicha habilidad es incremental, que tampoco resultó significativa.

Respecto a la relación entre las orientaciones de meta y los tipos de creencias, los resultados revelaron una correlación alta, positiva y significativa entre la creencia de que la habilidad es incremental y la orientación a la tarea, mientras que la relación también fue significativa entre dicha orientación a la tarea y la creencia de que la habilidad es estable, aunque en este caso la correlación fue moderada y negativa. Por otro lado, se encontró una correlación significativa y positiva entre la orientación al ego y la creencia de que la habilidad es una entidad estable, mientras que la asociación entre dicha orientación al ego y la concepción de que la habilidad está determinada por el aprendizaje y es mejorable no resultó significativa (Tabla 1).

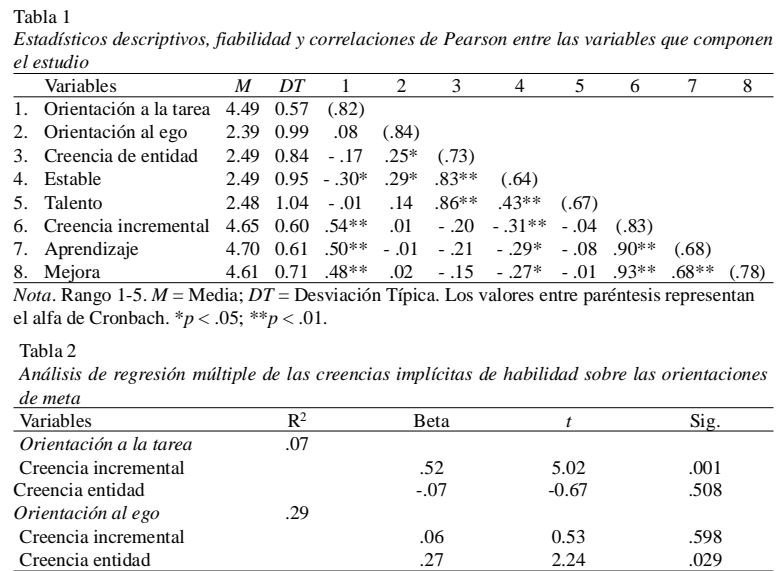

\section{Análisis de regresión}

Se analizó el poder predictivo de las creencias implícitas de habilidad (incremental y de entidad) sobre las orientaciones de meta (orientación al ego y orientación a la tarea).

Tal y como aparece en la Tabla 2, el análisis de regresión mostró que la creencia incremental de habilidad percibida predijo de forma positiva y significativa $\left(F_{(2,68)}=14.02, p<.001\right)$ la orientación a la tarea $(\beta=.52, p<.001)$, explicando un $29.2 \%$ de su varianza total. Por otra parte, la creencia de entidad sobre la habilidad deportiva predijo de manera positiva y significativa $\left(F_{(2,68)}=2.50, p<.05\right)$ la orientación al ego $(\beta=.27, p<.05)$, explicando el $7 \%$ de la varianza.

\section{Discusión}

El objetivo del presente estudio fue examinar las creencias implícitas sobre la habilidad y las orientaciones motivacionales en 71 jugadoras de sóftbol pertenecientes a los seis equipos de máximo nivel competitivo de México, teniendo en cuenta la edad, los años de experiencia y el equipo al que pertenece.

Los resultados sobre las diferencias en los tipos de creencias y las orientaciones de meta por grupos de edad, años de 
experiencia y el equipo al que pertenecen las deportistas no revelaron diferencias significativas. Esto indica que la concepción sobre la habilidad deportiva y el tipo de orientación que presentan las jugadoras de sóftbol que participaron en el presente estudio fue similar independientemente de la edad, de los años que llevan practicando su deporte y del equipo al que pertenecen. Hasta el momento, no hemos encontrado estudios que hayan analizado diferencias en función de estas variables en el contexto deportivo, no obstante, en la educación física, algunos estudios encontraron diferencias sobre la concepción de los estudiantes sobre sus habilidades a través de los diferentes cursos, donde aquellos con mayor edad presentaron una mayor creencia estable sobre su habilidad que sus similares de menor edad (Xiang \& Lee, 1998; Xiang, Lee \& Shen, 2001).

En relación a las variables de orden superior de las creencias implicitas, las jugadoras de sóftbol mostraron una creencia incremental superior que la creencia de entidad. Respecto a las variables de primer orden, las jugadoras informaron de una mayor concepción de que la habilidad es producto del aprendizaje y es mejorable en comparación con la creencia de que la habilidad es estable y producto del talento natural. En cuanto a las orientaciones de meta, las jugadoras mostraron una mayor orientación a la tarea que al ego, es decir, principalmente se comparan consigo mismas, se centran en el esfuerzo por aprender y dominar la tarea en lugar de compararse con otras jugadoras, y centrarse en hacerlo mejor que las demás para lograr un mayor reconocimiento, estatus social o ser más populares. Estos resultados son congruentes con los presentados por Sarrazin et al. (1996) en su estudio realizado con niños que participaban en algun deporte.

Los resultados del análisis de correlación entre las creencias sobre la habilidad deportiva y las orientaciones de meta fueron congruentes con los resultados del estudio de Sarrazin et al. (1996) y Fonseca y Paula Brito (2000) al encontrar correlaciones altas y positivas entre la creencia de que la habilidad deportiva es incremental, que está determinada por el aprendizaje y es mejorable con la orientación a la tarea. Al igual que en el estudio de King y Williams (1997), nuestros resultados revelaron que la creencia como entidad y la concepción de que la habilidad deportiva es una característica estable se correlacionó moderada y positivamente con la orientación al ego. Por otro lado, también se encontró una relación moderada y negativa entre la creencia de que la habilidad es estable y la orientación a la tarea, coincidiendo específicamente con los resultados con los obtenidos por Sarrazin et al. (1996) y King y Williams (1997). Esto indica que cuando más se crea que la habilidad deportiva es un atributo fijo e invariable menos orientado a la tarea se está para la práctica deportiva.

Por último, los resultados de la predicción de las orientaciones de meta por medio de las creencias implícitas fueron consistentes con los resultados del segundo estudio realizado por Biddle et al. (2003), ya que la creencia incremental predijo positivamente la orientación a la tarea, esto quiere decir que la concepción de que la habilidad deportiva es maleable predice la disposición de que el éxito significa superarse a sí mismo y lograr maestría en la tarea. Y por el contrario, la creencia de entidad predijo de manera positiva la orientación al ego, esto significa que la concepción de que la habilidad es un atributo fijo e invariable predice la orientación donde el éxito significa hacerlo mejor que los demás y tener mayor reconocimiento o estatus social. Los beneficios de estar orientado a la tarea han sido ampliamente estudiados en la literatura científica, incluyendo entre dichos beneficios una mayor diversión, persistencia, motivación y esfuerzo, así como menor ansiedad y conductas inmorales (Duda, 2005).

El presente estudio no está exento de limitaciones. Se trata de un estudio correlacional por lo que no se pueden realizar inferenciales causales entre las diferentes variables. Desde el punto de vista de los antecedentes de las metas de logro, se ha examinado un antecedente personal de la adquisición de las metas de logro en el contexto deportivo, no obstante, habría que introducir en estudios futuros la percepción del clima motivacional creado por el entrenador como otro antecedente importante en dicha adquisición. En este sentido, se ha encontrado que la forma en la que los entrenadores valoran la competencia en el contexto deportivo tiene sus implicaciones en las orientaciones de meta adoptadas por sus deportistas. En concreto, se ha encontrado que la percepción de un clima de implicación en la tarea actúa de predictor positivo de la orientación a la tarea, mientras que la percepción de un clima de implicación en el ego se ha manifestado como predictor positivo de una orientación al ego (e.g., Balaguer, Duda, Castillo, Moreno, \& Crespo, 2009; Balaguer, Castillo, Duda, \& García-Merita, 2011; Sánchez, Leo, Gómez, Sánchez, Cruz, \& García, 2009). Así pues, si queremos que nuestros deportistas adopten una orientación a la tarea por sus consecuencias importantes sobre la calidad de su experiencia, los entrenadores deberían crear climas de implicación en la tarea. Uno de los caminos a seguir podría ser la utilización de las estrategias del acrónimo TARGET (Diseño de la Tarea, fuente de Autoridad, naturaleza del Reconocimiento, forma de aGrupar, cómo se realiza la Evaluación y el Tiempo ofrecido para ejecutar las tareas; véase Ames, 1992; Balaguer, 2007) para que ellos aprendan a crear climas motivacionales positivos de entrenamiento. Actualmente ya existen ejemplos de intervención en la formación de entrenadores realizados en diversos países europeos que podrían servir de guía en futuros trabajos (véase por ejemplo, Castillo, Ramis, Cruz, \& Balaguer, 2015; Duda et al., 2013).

\section{Conclusiones}

Las jugadoras de sóftbol de élite consideran que la habilidad es producto del aprendizaje y es mejorable en comparación con la creencia de que la habilidad es estable y producto del talento natural. Asimismo, las jugadoras mostraron una mayor orientación a la tarea que al ego, es decir, principalmente se comparan consigo mismas, se centran en el esfuerzo por aprender y dominar la tarea en lugar de compararse con otras jugadoras, y centrarse en hacerlo mejor que las demás para lograr un mayor reconocimiento, estatus social o ser más populares.

Los resultados revelan que cuando más se crea que la habilidad deportiva está determinada por el aprendizaje y es mejorable más se está orientada a la tarea cuando se practica deporte, mientras que cuando se considerada que la habilidad deportiva es un atributo fijo e invariable menos orientada a la tarea se está para la práctica deportiva.

En definitiva, debemos fomentar que nuestros deportistas adopten una orientación a la tarea por sus consecuencias importantes sobre la calidad de su experiencia. La clave podría estar en formar a los entrenadores para que generen climas de implicación en la tarea en el contexto deportivo.

\section{Referencias}

Ames, C. (1992). Achievement goals, motivational climate, and motivational processes. En G. Roberts (Ed.), Motivation in sport and exercise (pp. 161-176). Champaing, IL: Human Kinetics.

Balaguer, I. (2007). Clima motivacional, calidad de la implicación y bienestar psicológico: una propuesta de intervención en equipos deportivos. En A. Blanco y J. Rodríguez Marín (Eds.), Intervención Psicosocial (pp. 135-162). Madrid: Pearson. Prentice Hall.

Balaguer, I., Castillo, I., Duda, J. L., \& García-Merita, M. L. 
(2011). Asociaciones entre la percepción del clima motivacional creado por el entrenador, orientaciones disposicionales de meta, formas de autorregulación y vitalidad subjetiva en jóvenes jugadoras de tenis. Revista de Psicología del Deporte, 20(1), 133-148.

Balaguer, I., Duda, J. L., Castillo, I., Moreno, Y., \& Crespo, M. (2009). Interacciones entre las perspectivas situacionales y disposicionales de meta y el burnout psicológico de los tenistas junior de la elite internacional. Acción Psicológica, 6(2), 63-75.

Biddle, S., Soos, I., \& Chatzisarantis, N. (1999), Predicting physical activity intentions using a goal perspectives approach: A study of Hungarian youth. Scandinavian Journal of Medicine and Science in Sports, 9, 353-357.

Biddle, S. J. H., Wang, C. K. J., Chatzisarantis, N. L. D., \& Spray, C. M. (2003). Motivation for physical activity in young people: entity and incremental beliefs about athletic ability. Journal of Sports Sciences, 21(12), 973-989.

Castillo, I., Ramis, Y., Cruz, J., \& Balaguer, I. (2015). Formación de Entrenadores de Fútbol Base en el proyecto PAPA. Revista de Psicología del Deporte, 24(1), 131-138.

Chin, N., Knoo, S., \& Low, W. (2012). Self-determination and goal orientation in track and field. Journal of Human Kinetics, 33, 151-161.

Cronbach, L. (1951). Coefficient alpha and the internal structure of tests. Psychometrika, 16(3), 297-334.

Duda, J. L. (1989). Relationship between task and ego orientation and the perceived purpose of sport among high school athletes. Journal of Sport \& Exercise Psychology, 11(3), 318-335.

Duda, J. L. (2005). Motivation in sport: The relevance of competence and achievement goals. In A. J. Elliot \& C. S. Dweck (Eds.), Handbook of competence and motivation (pp. 318-335). New York, NY: Guildford.

Duda, J. L. (2012). The coach-created motivational climate, youth athletes' well-being, and intentions to continue participation. Journal of Clinical Sport Psychology, 6, 166179.

Duda, J. L., Chi, L., Newton, M. L., Walling, M. D. \& Catley, D. (1995). Task and ego orientation and intrinsic motivation in sport. International Journal of Sport Psychology, 26(1), 40-63.

Duda, J. L., Quested, E., Haug, E., Samdal, O., Wold, B., Balaguer, I., ... Cruz, J. (2013). Promoting Adolescent health through an intervention aimed at improving the quality of their participation in Physical Activity (PAPA): background to the project and main trial protocol. International Journal of Sport and Exercise Psychology, 11, 319-327.

Duda, J.L. \& Whitehead, J. (1998). Measurement of goal perspectives in the physical domain. In J. L. Duda (Ed.), Advances in sport and exercise psychology measurement (pp. 21-48). Morgantown, WV: Fitness Information Technology.

Dweck, C. S. (1986). Motivational processes affecting learning. American psychologist, 41(10), 1040.

Dweck, C. S., \& Leggett, E. L. (1988). A social-cognitive approach to motivation and personality. Psychological review, 95(2), 256

Dweck, C. S., Chiu, C. Y., \& Hong, Y. Y. (1995). Implicit theories and their role in judgments and reactions: A word from two perspectives. Psychological inquiry, 6(4), 267-285.

Dweck, C. S. (1999). Self-theories: Their role in motivation, personality, and development. Philadelphia, PA: Taylor \& Francis.

Fonseca, A. M., \& Paula Brito, A. (2000). Las concepciones sobre la competencia deportiva y los objetivos de logro. Revista de Psicología del Deporte, 9(1-2), 159-176.
Hagger, M., \& Chatzisarantis, N. (2005). The Social Psychology of Exercise and Sport. Maidenhead, GBR: McGraw-Hill Education.

Jourden, F., Bandura, A., \& Banfield, J. T. (1991). The impact of conceptions of ability on selfregulatory factors and motor skill acquisition. Journal of Sport \& Exercise Psychology, 13, 213-226.

King, L. A., \& Williams, T. A. (1997). Goal orientation and performance in martial arts. Journal of Sport Behavior, 20, 397-411.

López-Walle, J., Balaguer, I., Meliá, J. L., Castillo, I., \& Tristán, J. (2011). Adaptación a la población mexicana del Cuestionario de Orientación al Ego y a la Tarea en el Deporte (TEOSQ). Revista de Psicología del Deporte, 20(2), 523536.

Nicholls, J. G. (1984). Achievement motivation: Conceptions of ability, subjective experience, task choice, and performance. Psychological Review, 91(3), 328-346.

Nicholls, J. (1989). The competitive ethos and democratic education. Cambridge, MA: Harvard University Press.

Nicholls, J. G. (1992). The general and the specific in the development and expression of achievement motivation. En G. C. Roberts (Ed.), Motivation in sport and exercise (pp. 31-56). Champaign, IL: Human Kinetics.

Ntoumanis, N., \& Biddle, S. J. (1999). A review of motivational climate in physical activity. Journal of Sports Sciences, 17(8), 643-665.

Moreno-Murcia, J. A., Cervelló-Gimeno, E. M., MartínezGalindo, M. C., \& Moreno, R., (2013). Validación de la Escala de Creencias Implícitas de habilidad (CNAAQ-2) al contexto español. Diferencias según la práctica físico-deportiva. Revista Internacional de Ciencias del Deporte, 32(9), 100-113.

Organización Mundial de la Salud (2010). Global recommendations on physical activity for health. Geneva. Extraído de

http://whqlibdoc.who.int/publications/2010/ 9789241599979_eng.pdf

Parish, L. E., \& Treasure, D. C. (2003). Physical activity and situational motivation in physical education: Influence of the motivational climate and perceived ability. Research Quarterly for Exercise and Sport, 74(2), 173-182.

Sánchez, P. A., Leo, F. M., Gómez, F. R., Sánchez, D., Cruz, E., \& García, T. (2009). Orientaciones de metas y clima motivacionales de los otros significativos en jóvenes de jugadores extremeños de balonmano. Retos. Nuevas tendencias en Educación Física, Deporte y Recreación, 16, 22-27.

Sarrazin, P., Biddle, S., K., Famose, J.-P., Cury, F., Fox, K., \& Durand, M. (1996). Goal orientations and conceptions of the nature of sport ability in children: A social cognitive approach. British Journal of Social Psychology, 35(3), 399414.

Standage, M., \& Treasure, D. C. (2002). Relationship among achievement goal orientations and multidimensional situational motivation in physical education. British Journal of Educational Psychology, 72(1), 87-103.

Van-Yperen, N. W., \& Duda, J. L. (1999). Goal orientations, beliefs about success, and performance improvement among young elite Dutch soccer players. Scandinavian Journal of Medicine \& Science in Sports, 9, 358-364.

Xiang, P., \& Lee, A. (1998). The development of self-perceptions of ability and achievement goals and their relations in physical education. Research Quarterly for Exercise and Sport, 69(3), 231-241.

Xiang, P., Lee, A., \& Shen, J. (2001). Conceptions of ability and achievement goals in physical education: Comparisons of American and Chinese students. Contemporary Educational Psychology, 26(3), 348-365. 